\title{
Drawing a different picture with pencil lead as matrix-assisted laser desorption/ionization matrix for fullerene derivatives
}

\author{
Leanne C Nye ${ }^{1}$, Hartmut Hungerbühler ${ }^{2}$ and Thomas Drewello ${ }^{3}$
}

\begin{abstract}
Inspired by reports on the use of pencil lead as a matrix-assisted laser desorption/ionization matrix, paving the way towards matrix-free matrix-assisted laser desorption/ionization, the present investigation evaluates its usage with organic fullerene derivatives. Currently, this class of compounds is best analysed using the electron transfer matrix trans-2-[3-(4-tertbutylphenyl)-2-methyl-2-propenylidene] malononitrile (DCTB), which was employed as the standard here. The suitability of pencil lead was additionally compared to direct (i.e. no matrix) laser desorption/ionization-mass spectrometry. The use of (DCTB) was identified as the by far gentler method, producing spectra with abundant molecular ion signals and much reduced fragmentation. Analytically, pencil lead was found to be ineffective as a matrix, however, appears to be an extremely easy and inexpensive method for producing sodium and potassium adducts.
\end{abstract}

\begin{abstract}
Keywords
Matrix-assisted laser desorption/ionization, pencil lead, (DCTB), sodium and potassium adducts, matrix, fullerene derivatives
\end{abstract}

Received 2 August 2017; accepted 12 October 2017

\section{Introduction}

The use of the material commonly referred to as 'the matrix' in matrix-assisted laser desorption/ionization (MALDI) is both a blessing and a curse. On the one hand, it delivers the softness for the overall desorption/ ionization steps leading to ions that may otherwise decompose and on the other, the detection of low molecular weight compounds may be obscured by ions derived from the matrix itself. Moreover, the procedure by which matrix and sample are applied jointly to the target is often time-consuming and laborious which is not advantageous for the development of high-throughput methodologies. As a consequence, there has been increasing interest in modifications of MALDI that would still show its softness but without the use of the matrix. Such matrix-free desorption/ionization approaches are frequently categorized as surface-assisted laser desorption/ionization (SALDI), whereby a modified surface has properties that effectively provide conditions for overall soft desorption and ionization. ${ }^{1-6}$ The sample can be applied directly onto the surface and direct laser desorption/ionization (LDI) may be applied. The ease of sample preparation, however, often comes after what can be laborious and/or expensive procedures to modify the surface, such as the etching of silicon with HF to produce porous silicon for desorption/ionization on silicon (DIOS). ${ }^{7}$

Langley and co-workers introduced an amazingly simple and inexpensive approach through the use of pencil lead, ${ }^{8,9}$ where the only surface modification is to draw a pencil over the stainless steel target plate. Pencil lead-MALDI combines two approaches within the modern development of MALDI. First, the ease of the target preparation provides an interesting addition to the repertoire of matrix-free MALDI or SALDI. ${ }^{1-6}$ Second, it features prominently amongst approaches that are applying carbon-based nano-materials such as graphite, ${ }^{10,11}$ fullerene derivatives, ${ }^{12,13}$ carbon nanotubes, ${ }^{14}$-horns, ${ }^{15}$ as well as other carbon-based

\footnotetext{
${ }^{1}$ Department of Surgery and Cancer, Imperial College, London, UK ${ }^{2}$ Beuth Hochschule für Technik Berlin, Fachbereich II, Berlin, Germany ${ }^{3}$ Physical Chemistry I, Department of Chemistry and Pharmacy, University of Erlangen-Nuremberg, Erlangen, Germany
}

\section{Corresponding author:}

Thomas Drewello, Physical Chemistry I, Department of Chemistry and Pharmacy, University of Erlangen-Nuremberg, Egerlandstrasse 3, 91058 Erlangen, Germany.

Email: thomas.drewello@fau.de 
nanostructured materials ${ }^{5,6}$ and the carbon allotrope that became available most recently: graphene. ${ }^{16}$

The component of pencil lead assumed to provide the matrix effect is graphite, which has previously been successfully employed as a MALDI matrix. ${ }^{10,11}$ Issues arise, however, as it is typically difficult to apply to the target plate as well as to achieve an even reproducible surface coverage. Generally the softer the pencil, the more graphite it contains. There are many reasons why pencil lead would be a desirable matrix ${ }^{8,9}$ - its usage is safe, cheap with a quick and easy preparation, there are no solvent compatibility issues and the hydrophobic surface tends to help in producing a sweet spot.

In their initial paper, ${ }^{8}$ Langley and co-workers applied pencil lead to the analysis of various samples including peptides such as substance $\mathrm{P}$, polymers, actinide metals such as uranium and terfenadine. For this study, a range of pencil grades were tested, ranging from $4 \mathrm{H}$ to $6 \mathrm{~B}$. It was found that $6 \mathrm{~B}$ gave the best performance and this was assumed to be caused by it having the highest graphite content of all the pencils tested. Moreover, the softness of that particular pencil led to an easier application with more even coverage and appeared to offer a better reproducibility. A subsequent study ${ }^{9}$ tested a wider range of pencils on a selection of 50 small molecules, which included peptides, polymers, steroids and sugars. Contrary to the previous study, ${ }^{8}$ the $2 \mathrm{~B}$ pencil was found to give the best results for the range of samples tested.

Pencil lead-MALDI has also been applied successfully to polymers, specifically to silyl hydride functionalised polystyrenes, which are particularly sensitive to the preparation methods commonly used in MALDI for polymers. ${ }^{17}$ For instance, the common use of dithranol as the matrix, with silver trifluoroacetate (AgTFA) as an additive, led to oxidation of the silane moiety. It was found that by using pencil lead and LiTFA, there were no side reactions, and the only adducts formed were lithium adducts. For pencil leadMALDI of long alkyl esters of succinic and maleic acid, significant improvements in both reproducibility and sensitivity have been reported. ${ }^{18}$ Pencil lead-MALDI analysis of model peptides has been compared with other matrix-free targets and gave only moderate performance. ${ }^{19}$ Also, the use of colloidal graphite led to improved results compared to the one of pencil lead for the analysis of low molecular weight analytes. ${ }^{20}$

The fullerene derivatives under investigation are displayed in Figure 1. These methanofullerenes were produced by cyclopropanation ${ }^{21,22}$ and belong to a most versatile class of fullerene derivatives which gained tremendous importance as a means to induce structural changes and to modify material properties. ${ }^{23}$ These adducts of $\mathrm{C}_{60}$ with bis(alkoxycarbonyl)methylenes have been the subject to an earlier direct LDI study in which they produced intact molecular ions but also showed a considerable degree of fragmentation. ${ }^{24}$ This makes them interesting candidates to test the performance of a MALDI matrix, which should enhance the molecular ion abundance and reduce fragmentation. We have used these analytes to establish the superiority of the trans-2-[3-(4-tert-butylphenyl)-2-methyl-2-propenylidene] malononitrile (DCTB) matrix in comparison with other organic matrix materials. ${ }^{25}$ In addition to elucidating the electron transfer mechanism using the DCTB matrix, ${ }^{26,27}$ we have successfully employed DCTB to a variety of fullerene-related MALDI studies. $^{28-32}$

\section{Experimental}

All $\mathrm{C}_{60}$ adducts (Figure 1) were synthesized according to literature procedures ${ }^{21}$ and purified by chromatographic means, so that individual compounds were free of any ligand attainment other than indicated by their formula.

All analytes were dissolved in toluene (Fisher Chemicals, 99\% laboratory grade) at a $1 \mathrm{mg} / \mathrm{ml}$ concentration. A Fisherbrand Whirlimixer was used to ensure complete dissolution. DCTB (Fluka, Sigma-Aldrich, 99\% HPLC grade) was prepared in a $10 \mathrm{mg} / \mathrm{ml}$ solution in toluene, which was added to the sample as required, typically in a 1:50 sample:DCTB molar ratio. $6 \mathrm{~B}$ and 8B Staedtler branded Mars Lumograph pencils were

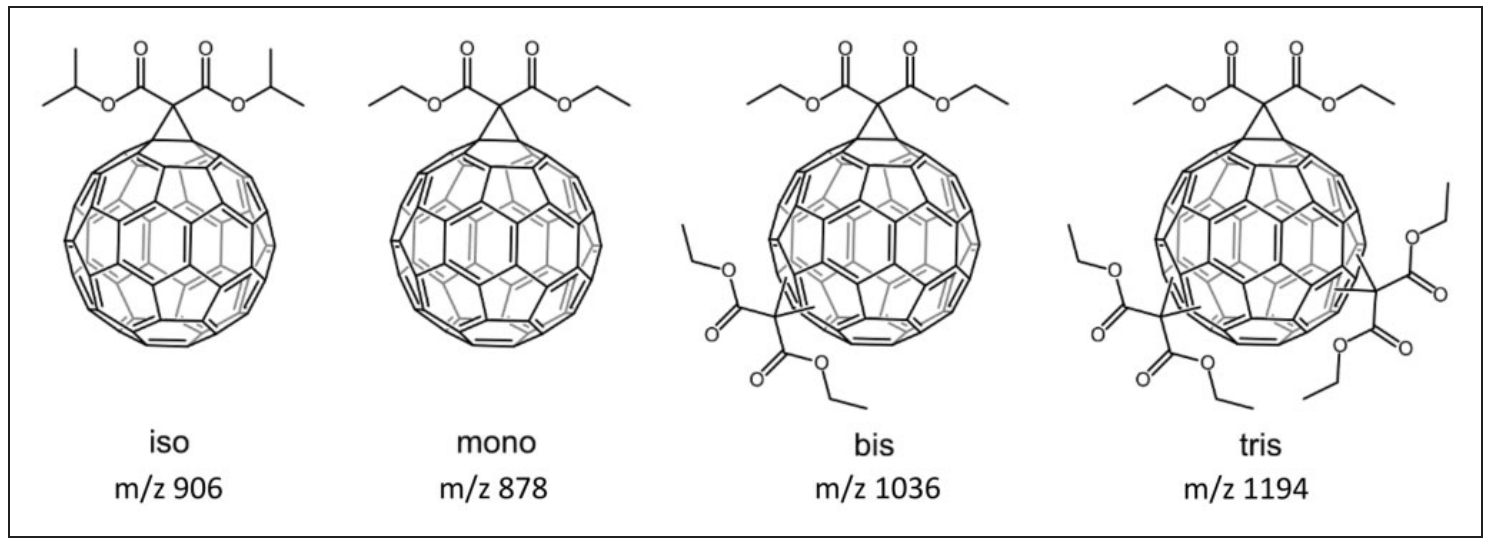

Figure 1. Structures of the fullerene derivatives under investigation with abbreviated names and nominal masses. 
used throughout and were applied individually on the target spots. A pressure equivalent to writing was used to transfer pencil lead to the target plate, except in the experiments otherwise stated. The entire slide was held in an air stream to remove any loose pencil lead prior to sample application. Experiments in which higher pencil lead loadings were tested gave no other results.

The sample solutions were applied one drop at a time using a 1-10 $\mu$ l Barky mikroliterpipette and allowed to dry between applications. Typically $5 \mu \mathrm{l}$ of the solution was applied to each target spot.

For the DCTB experiments, the DCTB solution and sample were thoroughly mixed and applied together, typically $5 \mu \mathrm{l}$ of this solution was used per target spot. Mass spectra were acquired with a Bruker Ultraflex III. This MALDI-ToF (time-of-flight) mass spectrometer comes equipped with a SmartBeam ${ }^{\mathrm{TM}}$ laser which operates at $355 \mathrm{~nm}$, with a repetition rate of between 1 and $200 \mathrm{~Hz}$. Spectra were exported from the Bruker FlexAnalysis software for presentation purposes into the Origin software package.

\section{Results and discussion}

The adduct of $\mathrm{C}_{60}$ with bis(isopropoxycarbonyl)methylene (Figure 1) will be referred to in the following as iso and the corresponding adducts with one, two and three bis(ethoxycarbonyl)-methylene ligands as mono, bis and tris, respectively. All four investigated methanofullerenes showed a similar behaviour under (MA)LDI conditions. In most experiments, iso was used because it is the most labile derivative of the four, which makes it the ideal candidate to test the softness of a method. However, to introduce the typical appearance of the pencil lead-MALDI spectra, bis was used as the analyte in Figure 2, as it can decompose not only into $\mathrm{C}_{60}$ but also into the mono-ligated species.

Figure 2 compares two pencil lead-MALDI spectra of bis using 6B (Figure 2(a)) and 8B pencil (Figure 2(b)). The spectra were obtained at a laser fluence adjusted to produce spectra with an overall high signal-to-noise ratio, rather than adjusting at the threshold of ion formation. Both spectra show extensive fragmentation leading to $\mathrm{C}_{60}{ }^{+}$as the dominant peak at $\mathrm{m} / \mathrm{z}$ 720. There are also $\left[\mathrm{C}_{61} \mathrm{H}\right]^{+}$fragment ions at $\mathrm{m} / \mathrm{z} 733$ and to a lesser extent a signal that would correspond with the mass of mono is observed at $\mathrm{m} / \mathrm{z} 878$. As all the compounds were carefully purified prior to the experiment, there can be no doubt that the signals relating to $\mathrm{C}_{60}$ and mono are caused by fragmentation of bis. However, there is also formation of the molecular ion at m/z 1036 and as reported a typical feature in previous pencil lead-MALDI research, the quasi-molecular ions are abundantly formed as the

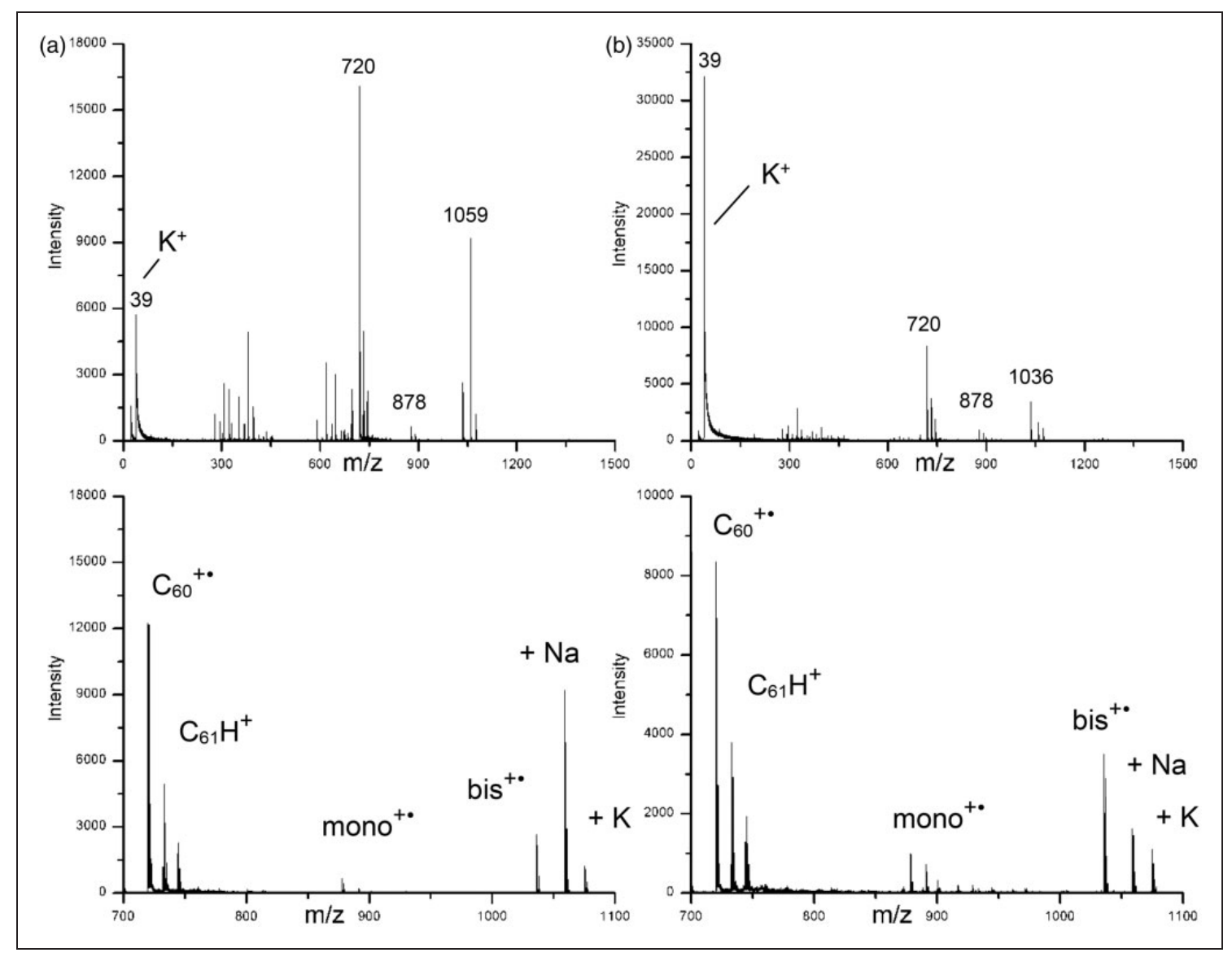

Figure 2. Pencil lead-MALDI mass spectra of bis applying (a) $6 \mathrm{~B}$ pencil and (b) $8 \mathrm{~B}$ pencil. Top line - full $\mathrm{m} / \mathrm{z}$ range measured, bottom line - from $\mathrm{m} / \mathrm{z}$ 700-1100. 


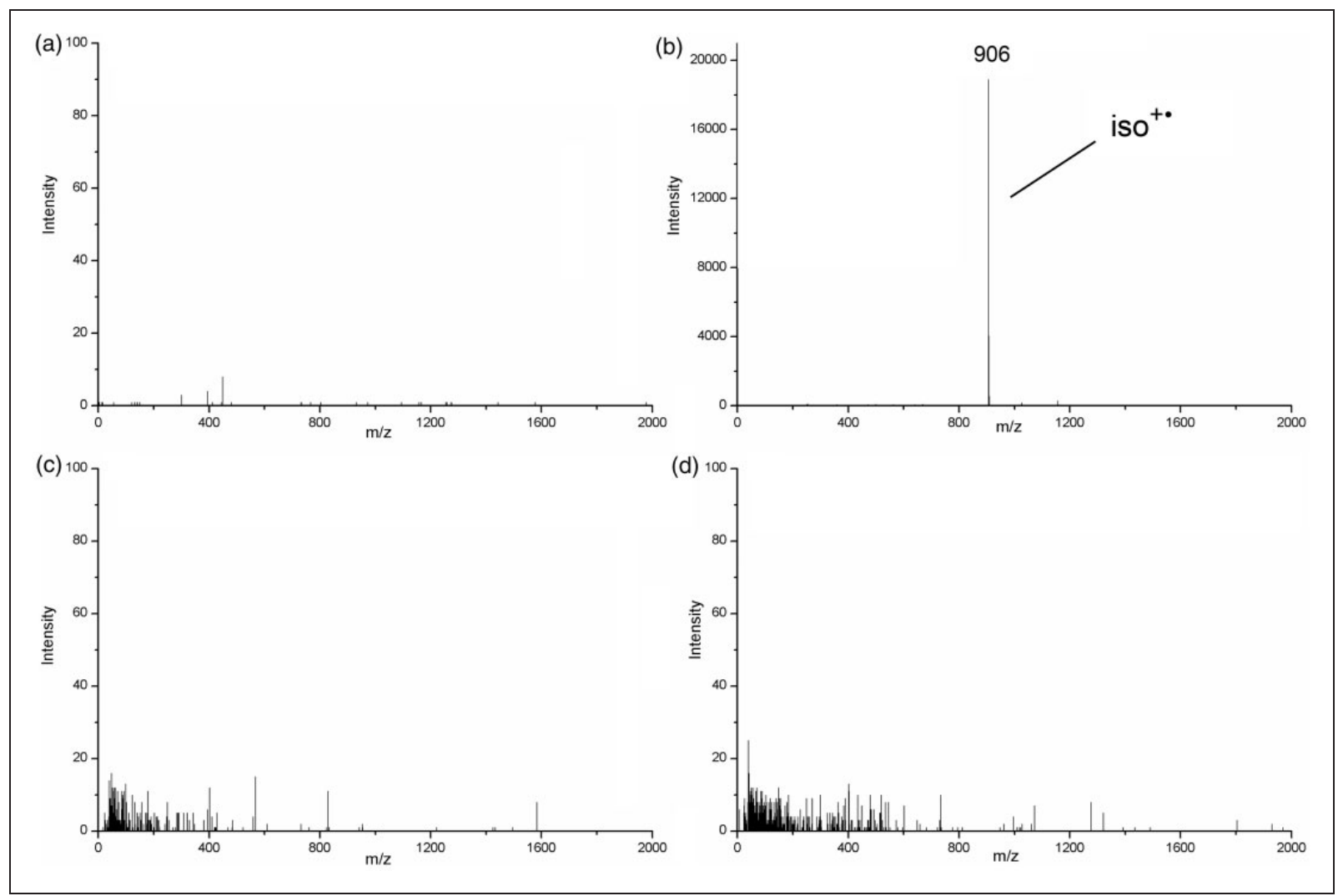

Figure 3. Mass spectra of iso obtained at $8 \%$ laser fluence using (a) LDI, (b) DCTB-MALDI, (c) 6B pencil lead-MALDI and (d) $8 B$ pencil lead-MALDI.

result of sodium and potassium addition to the intact compound. 6B pencil lead-MALDI (Figure 2(a)) shows the sodiated molecular ion peak as the most intense of the three at $\mathrm{m} / \mathrm{z} 1059$. Utilising the $8 \mathrm{~B}$ pencil (Figure 2(b)), the potassium peak at $\mathrm{m} / \mathrm{z} 39$ dominates the spectrum. Direct comparison of the ion abundances in both spectra reveals how much more abundant the potassium production is from the $8 \mathrm{~B}$ pencil with approx. 32,000 counts compared to $6 \mathrm{~B}$ with 6000 counts. However, the $6 \mathrm{~B}$ pencil appears to produce more analyte-derived ions (that is $\mathrm{C}_{60}$-containing) than $8 \mathrm{~B}$.

In terms of matrix protection, pencil lead does not appear to offer any. In fact, the pencil lead-MALDI spectra are very similar in appearance to those obtained using direct LDI (see below). This was the case for all four fullerene derivatives analysed. When this method is compared to spectra obtained using DCTB as a matrix for MALDI-mass spectrometry (MS) (see below), it becomes apparent that DCTB offers a far superior protection for this class of compounds. However, pencil lead appears to aid ionization through the addition of sodium and potassium.

The sodiated and potassiated quasi-molecular ions clearly originate from the presence of pencil lead on the target, as the result of $\mathrm{Na}^{+}$and $\mathrm{K}^{+}$attachment to the neutral molecule. All other non-metallated ions, such as $\mathrm{C}_{60}{ }^{+}$, the mono ${ }^{+}$fragment ion and the bis ${ }^{+}$. radical cation can be assumed to result from direct LDI. This can be straightforwardly based on the close similarity of the direct LDI mass spectra and those obtained by pencil lead-MALDI. However, we cannot rule out contributions from electron transfer with ions derived from the pencil lead. The metallated quasi-molecular ions are not likely to contribute (through fragmentation) to any of the non-metallated ions, because of the low ionization potential of the alkali cations preventing charge transfer reactions. Unfortunately this assumption could not be checked in LIFT (MS/MS following pencil lead-MALDI) experiments of the molecular and quasi-molecular ions, as these were always too low abundant to perform such experiments.

Finally, the malonate ligand is confirmed as the location of the $\mathrm{Na}^{+} / \mathrm{K}^{+}$attachment, as pure $\mathrm{C}_{60}$ did not show signs of metalation in pencil lead-MALDI.

To thoroughly compare the performance of pencil lead-MALDI with DCTB-MALDI and direct LDI, the iso compound was analysed with each of those methods in the course of one session (using one target plate for all four methods), to negate fluctuations in machine performance. A series of spectra was obtained for each of the methods over a range of increasing laser fluences. The laser fluence is expressed here as an arbitrary unit in percent and ranged from 4 to $24 \%$, at $4 \%$ increments. Below $4 \%$, it was difficult to obtain a signal for any method and above $24 \%$, the spectra did not alter much with higher laser fluences.

Figure 3 displays the analysis of iso by direct LDI (Figure 3(a)), DCTB-MALDI (Figure 3(b)) and pencil 


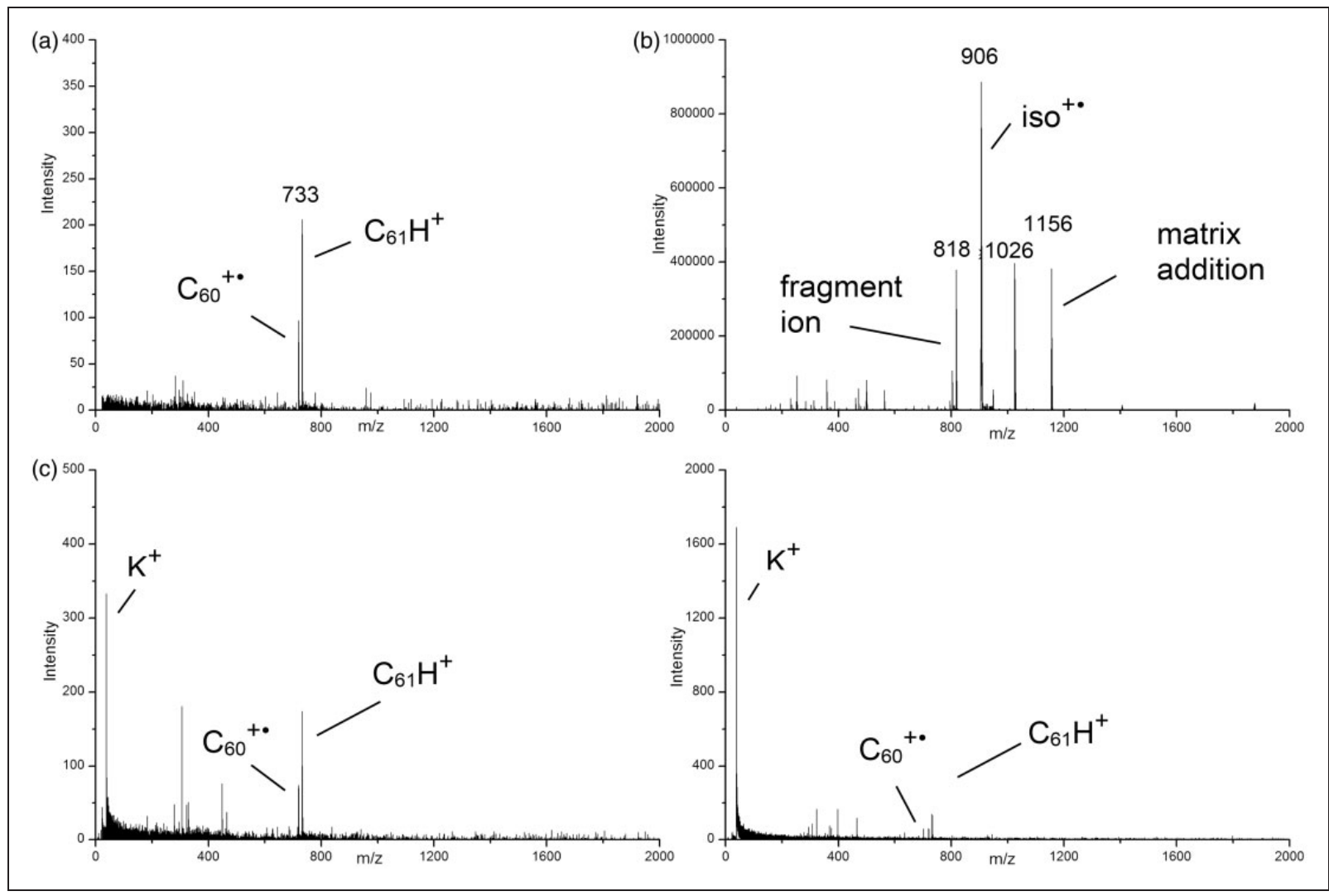

Figure 4. Mass spectra of iso obtained at $16 \%$ laser fluence using (a) LDI, (b) DCTB-MALDI, (c) $6 B$ pencil lead-MALDI and (d) $8 B$ pencil lead-MALDI.

lead-MALDI with 6B (Figure 3(c)) and 8B pencil (Figure 3(d)) at $8 \%$ laser fluence, the lowest fluence at which ions could be detected at all by one of the methods.

The $y$-axis displays actual intensities (counts), rather than relative intensities as percentages normalized to the most dominant peak. This allows for direct comparison between each method. In the LDI (Figure 3(a)), 6B (Figure 3(c)) and 8B (Figure 3(d)) pencil spectra, the peaks are not higher than 20 counts. Comparison to the DCTB-MALDI spectrum (Figure 3(b)), where the true molecular ion peak of iso is obtained at a value of approximately 19,000 counts, shows the effectiveness of the DCTB-MALDI performance. DCTB produces the molecular ion peak at very low laser fluences, thus reduces the threshold for ionization dramatically.

Figure 4 reveals that the onset of the ion formation for the LDI (Figure 4(a)), 6B (Figure 4(c)) and 8B pencil lead (Figure 4(d)) conditions coincides with a laser fluence of $16 \%$. The only analyte-derived ions with peaks at $\mathrm{m} / \mathrm{z} 720$ and $\mathrm{m} / \mathrm{z} 733$ are fragment ions as previously identified. Fragment ions may either arise from complete decomposition of directly ionised iso or through ionisation of fragments that result from decomposition of neutral iso. In any case, these ions indicate that fairly drastic conditions prevail. In the DCTB-MALDI spectrum (Figure 4(b)), the molecular ion is still the base peak, although additional signals appear. The peaks at lower masses than $\mathrm{m} / \mathrm{z}$ 906 may result from fragmentation (besides those derived from the matrix) and signals at higher mass can be attributed to the addition of DCTB to the analyte molecule. However, important is, that the fragmentation down to $\mathrm{C}_{60}{ }^{+} \bullet$ is still rather minute with the molecular analyte ion representing the most abundant peak.

With the further increase in laser fluence to $20 \%$ (Figure 5), low abundant peaks due to the radical cation and the sodiated and potassiated analyte molecule start to appear in the pencil lead-MALDI spectra (Figure 5(c) and (d)). These peaks are much lower in intensity when compared to the fragmentation peaks. The LDI mass spectrum (Figure 5(a)) is still only producing fragmentation peaks. For the DCTB-MALDI mass spectrum (Figure 5(b)), although the matrix and fragmentation peaks gain in abundance, the molecular ion peak is still the dominant signal. The overall ion abundance with DCTB-MALDI is orders of magnitude larger than with the other methods, which demonstrates the enhanced efficiency of desorption and soft ion formation of this approach.

With regard to the use of pencil lead as a matrix for the analysis of derivatised fullerenes, the present experiments have demonstrated that it is an unsuitable replacement for the current standard matrix DCTB. The formation of quasi-molecular ions is observed in the form of sodium and potassium adducts to 

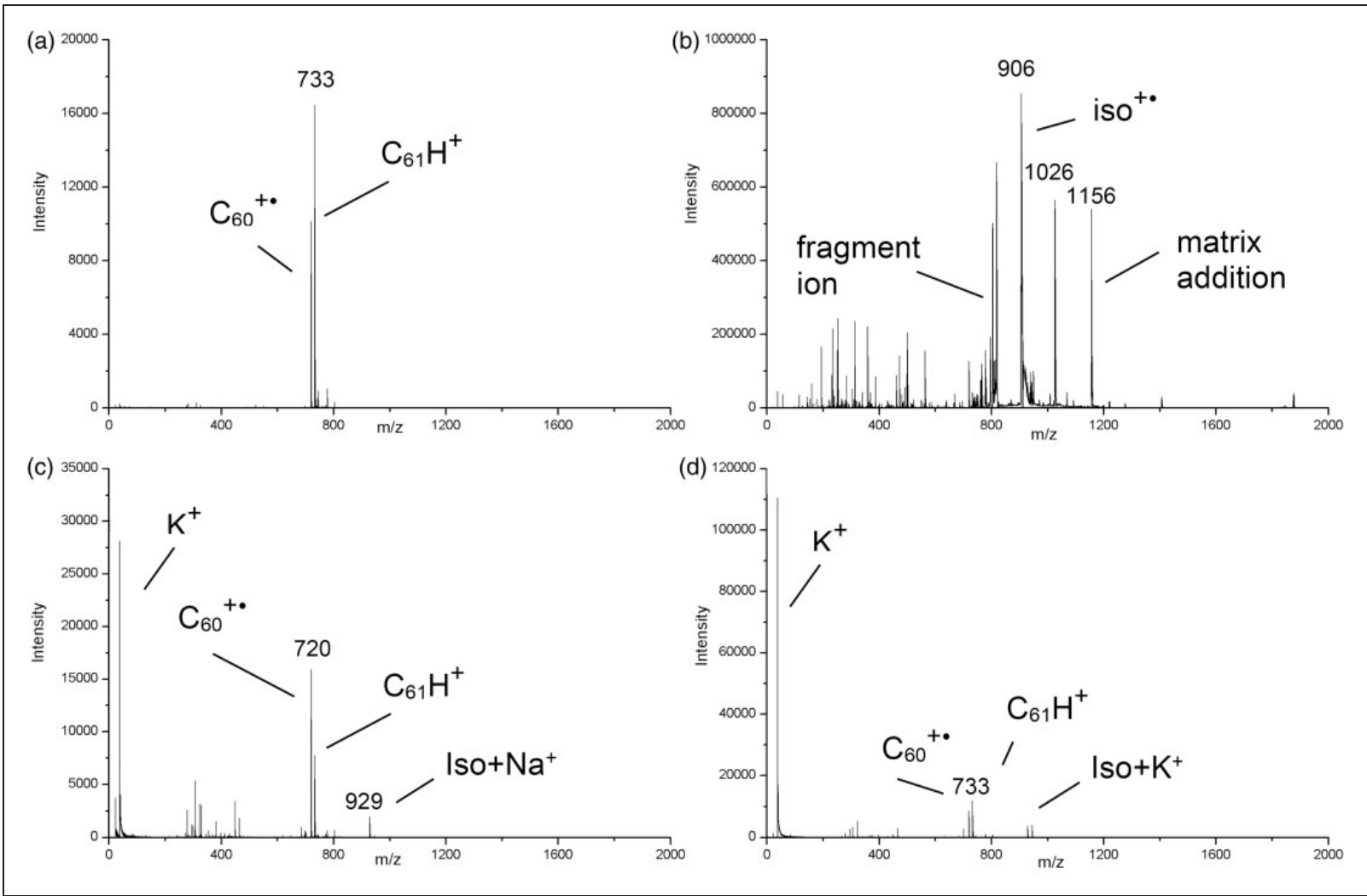

Figure 5. Mass spectra of iso obtained at $20 \%$ laser fluence using (a) LDI, (b) DCTB-MALDI, (c) $6 B$ pencil lead-MALDI and (d) $8 B$ pencil lead-MALDI.

the analyte. However, the formation of such quasimolecular ions occurs at such enhanced laser fluences that severe fragmentation is always observed as well. There is also some molecular ion present, however, the production of which is rather minute. Fragmentation is already abundantly observed at lower laser fluences than needed to observe the quasimolecular ions. Therefore, a large fraction of the fragmentation may be caused by direct laser activation of analyte that remains unprotected through the pencil lead matrix. DCTB comes out as a far superior matrix, producing a molecular ion peak at low laser fluences, with much reduced or no fragmentation at all. LDI is the worst performing method of the four in terms of molecular ion formation.

Fullerene derivatization is often unspecific leading to multiple addition of ligands to $\mathrm{C}_{60}$. A quick and reliable method to probe the product distribution is therefore desirable. In this context, pencil lead-MALDI was tested for the analysis of a 1:1:1 equi-molecular mix of mono, bis and tris, simulating a raw product following synthesis. The resulting spectra are shown in Figure 6 using $6 \mathrm{~B}$ and $8 \mathrm{~B}$ pencil. Certainly, since the performance of pencil lead-MALDI for the analysis of the isolated compound is rather poor, one cannot expect surprises for the analysis of a mixture.

But the outcome is even worse than expected. First, the huge signal for $\mathrm{C}_{60}$ is caused by extensive fragmentation. Second, the ratios of sodium and potassium addition are different between the compounds - apparently random. Mono appears to not form a great number of either sodium or potassium adducts. On $8 \mathrm{~B}$ pencil, bis shows the cation radical as the most abundant of the molecular ions (Figure 6(b)), yet it is the sodium adduct that is the most intense on 6B (Figure 6(a)). The most intense molecular ion for tris is the sodium adduct on both types of pencil, with a massive increase in the formation of the potassium adduct on 8B pencil (Figure 6(b)). Evidently, the spectra reflect the severe dissociation caused by direct LDI of the compounds with pencil lead failing to protect. Moreover, the different desorption efficiencies of the compounds may contribute as well as the fact that the malonate groups will statistically prefer the metal cation attainment in the order tris $>$ bis $>$ mono.

\section{Conclusion}

Pencil lead has been shown to be an unsatisfactory matrix for fullerenes and their derivatives, especially when compared to the benchmark of DCTB-MALDI. There is extensive fragmentation and the production of the molecular ion peak is commonly of low intensity. In general, higher laser fluences are needed than in DCTBMALDI to produce any ions. Analytically valuable is 

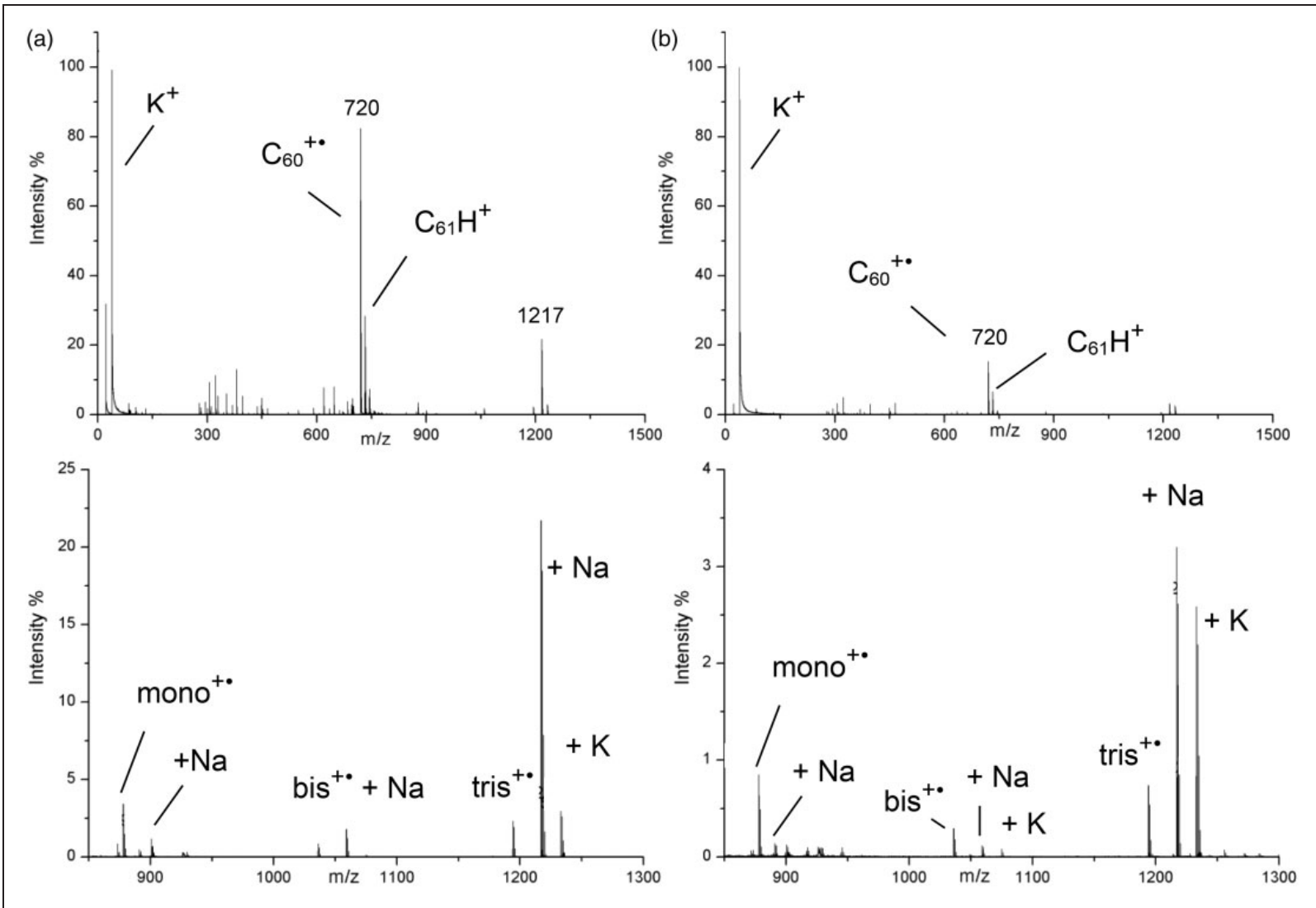

Figure 6. Mass spectra of mono: bis: tris in a 1:1:1 ratio, analysed by (a) $6 B$ pencil lead-MALDI and (b) 8B pencil lead-MALDI. Top line displays the full $\mathrm{m} / \mathrm{z}$ range of spectrum. The bottom line displays the molecular ion $\mathrm{m} / \mathrm{z}$ range.

the addition of sodium and potassium cations to analytes with heteroatoms. This could be used for identification, but also can be useful as a means to produce such adducts for further experiments. The difference between $6 \mathrm{~B}$ and $8 \mathrm{~B}$ pencil was not pronounced in terms of matrix performance, however, $8 \mathrm{~B}$ spectra produced higher quantities of potassium addition. Use of the $8 \mathrm{~B}$ pencil did also seem to hinder ion production occasionally, with often lower abundance of analytederived ions and resulting in lower quality spectra overall.

In summary, pencil lead-MALDI does not match the quality of DCTB-MALDI regarding softness, cleanliness of spectra and sensitivity, but could have its merits as a facile approach to generate sodium and potassium adducts of structurally suited fullerene derivatives.

\section{Acknowledgements}

LCN and TD are privileged to have worked with Peter Derrick for a long enough period of time to call him a colleague, mentor and friend. He is sadly missed in a world that is not the same without him. TD gratefully acknowledges funding form the Deutsche Forschungsgemeinschaft (DFG) - SFB 953 'Synthetic Carbon Allotropes'. We thank M Minameyer and $\mathbf{J}$ Hitzenberger for their help with the preparation of the manuscript.

\section{Dedication}

This article is dedicated to Prof. Peter J Derrick for the time at Warwick and beyond.

\section{Declaration of conflicting interests}

The author(s) declared no potential conflicts of interest with respect to the research, authorship, and/or publication of this article.

\section{Funding}

The author(s) disclosed receipt of the following financial support for the research, authorship and/or publication of this article: TD gratefully acknowledges funding form the Deutsche Forschungsgemeinschaft (DFG) - SFB 953 'Synthetic Carbon Allotropes'.

\section{References}

1. Guinan T, Kirkbride P, Pigou PE, et al. Surface-assisted laser desorption ionization mass spectrometry techniques for application in forensics. Mass Spectrom Rev 2015; 34: 627.

2. Rainer M, Qureshi MN and Bonn GK. Matrix-free and material-enhanced laser desorption/ionization mass spectrometry for the analysis of low molecular weight compounds. Anal Bioanal Chem 2011; 400: 2281. 
3. Kuzema PA. Small-molecule analysis by surface-assisted laser desorption/ionization mass spectrometry. $J$ Anal Chem 2011; 66: 1227.

4. Peterson DS. Matrix-free methods for laser desorption/ ionization mass spectrometry. Mass Spectrom Rev 2007; 26: 19.

5. Najam-ul-Haq M, Rainer M, Szabo Z, et al. Role of carbon nano-materials in the analysis of biological materials by laser desorption/ionization-mass spectrometry. J Biochem Biophys Meth 2007; 70: 319.

6. Coffinier Y, Boukherroub R and Szunerits S. Carbonbased nanostructures for matrix-free mass spectrometry. In: Yang N, Jiang X and Pang DW (eds) Carbon nanoparticles and nanostructures. Cham: Springer International Publishing, 2016, p.331.

7. Lewis WG, Shen Z, Finn MG, et al. Desorption/ionization on silicon (DIOS) mass spectrometry: background and applications. Int J Mass Spectrom 2003; 226: 107.

8. Black C, Poile C, Langley J, et al. The use of pencil lead as a matrix and calibrant for matrix-assisted laser desorption/ionisation. Rapid Commun Mass Spectrom 2006; 20: 1053.

9. Langley GJ, Herniman JM and Townell MS. 2B or not $2 \mathrm{~B}$, that is the question: further investigations into the use of pencil as a matrix for matrix-assisted laser desorption/ ionisation. Rapid Commun Mass Spectrom 2007; 21: 180.

10. Dale MJ, Knochenmuss R and Zenobi R. Graphite/ liquid mixed matrices for laser desorption/ionization mass spectrometry. Anal Chem 1996; 68: 3321.

11. Sunner J, Dratz E and Chen YC. Graphite surfaceassisted laser desorption/ionization time-of-flight mass spectrometry of peptides and proteines from liquid solutions. Anal Chem 1995; 67: 4335.

12. Hopwood FG, Michalak L, Alderdice DS, et al. C60assisted laser desorption/ionization mass spectrometry in the analysis of phosphotungstic acid. Rapid Commun Mass Spectrom 1994; 8: 881.

13. Shiea J, huang JP, Teng CF, et al. The use of watersoluble fullerene derivative as precipitating reagent and matrix-assisted laser desorption/ionization matrix to selectively detect charged species in aqueous solutions. Anal Chem 2003; 75: 3587.

14. $\mathrm{Xu} \mathrm{S}, \mathrm{Li} \mathrm{Y}$, Zou H, et al. Carbon nanotubes as assisted matrix for laser desorption/ionization time-of-flight mass spectrometry. Anal Chem 2003; 75: 6191.

15. Traldi P, Calandra E, Crotti S, et al. Matrix-assisted laser desorption/ionization, nanostructure-assisted laser desorption/ionization and carbon nanohorns in the dectection of antineoplastic drugs. 1. The case of irinotecan, sunitinib and 6-alpha-hydroxy paclitaxel. Eur J Mass Spectrom 2014; 20: 445.

16. Dong X, Cheng J, Li J, et al. Graphene as a novel matrix for the analysis of small molecules by MALDI-TOF MS. Anal Chem 2010; 82: 6208.

17. Berger-Nicoletti E, Wurm F, Kilbinger AFM, et al. Pencil lead as a matrix for MALDI-ToF mass spectrometry of sensitive functional polymers. Macromolecules 2007; 40: 746.
18. Kim J, Han SP, Kim J, et al. Detection of long alkyl esters of succinic and maleic acid using TLC-MALDI MS. Bull Kor Chem Soc 2011; 32: 915.

19. Shenar N, Cantel S, Martinez J, et al. Comparison of inert supports in laser desorption/ionization mass spectrometry of peptides: pencil lead, porous silica gel, DIOSchip and NALDI ${ }^{\mathrm{TM}}$ target. Rapid Commun Mass Spectrom 2009; 23: 2371.

20. Warren AD, Conway U, Arthur CJ, et al. Investigation of colloidal graphite as a matrix for matrix-assisted laser desorption/ionisation mass spectrometry of low molecular weight analytes. J Mass Spectrom 2016; 51: 491.

21. Hirsch A, Lamparth I and Karfunkel HR. Fullerene chemistry in three dimensions: isolation of seven regioisomeric bisadducts and chiral trisadducts of $\mathrm{C}_{60}$ and di(ethoxycarbonyl)methylene. Angew Chem Int Ed Engl 1994; 33: 437.

22. Hirsch A, Lamparth I, Grösser T, et al. Regiochemistry of multiple additions to the fullerene core: synthesis of a Th-symmetric hexacisadduct of C60 with bis(ethoxycarbonyl)methylene. J Am Chem Soc 1994; 116: 9385.

23. Hirsch A and Brettreich M. Fullerenes: Chemistry and reactions. Weinheim: Wiley-VCH Verlag, 2004.

24. Beck RD, Weis P, Hirsch A, et al. Laser desorption mass spectrometry of fullerene derivatives: laser-induced fragmentation and coalescence reactions. J Phys Chem 1994; 98: 9683.

25. Brown T, Clipston NL, Simjee N, et al. Matrix-assisted laser desorption/ionization of amphiphilic fullerene derivatives. Int J Mass Spectrom 2001; 210/211: 249.

26. Streletskii AV, Ioffe IN, Kotsiris SG, et al. In-plume thermodynamics of the MALDI generation of fluorofullerene anions. $J$ Phys Chem A 2005; 109: 714.

27. Vasil'ev YV, Khvostenko OG, Streletskii AV, et al. Electron transfer reactivity in matrix-assisted laser desorption/ionization (MALDI): ionization energy, electron affinity and performance of the DCTB matrix within the thermochemical framework. J Phys Chem A 2006; 110: 5967.

28. Vasil'ev VY, Kotsiris SG, Bashkin IO, et al. Bulk Produktion of a strong covalently linked $\left(\mathrm{C}_{60} \mathrm{H}_{\mathrm{x}}\right)_{2}$ dimer. J Phys Chem B 2005; 109: 11875.

29. Fati D, Leeman V, Vasil'ev YV, et al. Alkali cation attachment to derivatized fullerenes studied by matrixassisted laser desorption/ionization. J Am Soc Mass Spectrom 2002; 13: 1448.

30. Fati D, Vasil'ev YV, Wachter NK, et al. MALDI mass spectrometric determination of molecular site-specific charge localisation, and laser-induced coalescence reactivity of fullerenodendrimers. Int J Mass Spectrom 2003; 229: 3 .

31. Nye LC, Tzirakis MD, Orfanopoulos $\mathrm{M}$, et al. $\mathrm{C}_{60} \mathrm{H}_{2}$ : Recover and decline - an investigation by MALDI mass spectrometry. Chem Phys Lett 2010; 499: 209.

32. Kotsiris SG, Vasil'ev YV, Streletskii AV, et al. Application and evaluation of solvent-free matrixassisted laser desorption/ionization mass spectrometry for the analysis of derivatisezed fullerenes. Eur $J$ Mass Spectrom 2006; 12: 397. 